\title{
Dynamical approach to isotopic-distribution of fission fragments from actinide nuclei
}

\author{
Chikako Ishizuka ${ }^{1, a}$, Satoshi Chiba ${ }^{1}$, Alexander V. Karpov² and Yoshihiro Aritomo ${ }^{3}$ \\ ${ }^{1}$ Research Laboratory for Nuclear Reactors, Tokyo Institute of Technology, Tokyo, Japan \\ ${ }^{2}$ Joint Institute for Nuclear Research, Dubna, Russia \\ ${ }^{3}$ Kindai University, Osaka, Japan
}

\begin{abstract}
Measurements of the isotope distribution of fission fragments, often denoted as the primary fission yield (pre-neutron yield) or independent fission yield (post-neutron yield) are still challenging at low excitation energies, so that it is important to investigate it within a theory. Such quantities are vital for applications as well. In this study, fragment distributions from the fission of $U$ isotopes at low excitation energies are studied using a dynamical model. The potential energy surface is derived from the two center shell model including the shell and pairing corrections. In order to calculate the charge distribution of fission fragments, we introduce a new parameter $\eta_{Z}$ as the charge asymmetry, in addition to three parameters describing a nuclear shape, $z$ as the distance between two centers of mass, $\delta$ as fragment deformation, and $\eta_{A}$ as the mass asymmetry. Using this model, we calculated the isotopic distribution of ${ }^{236} U$ for the $n$-induced process ${ }^{235} U+n \rightarrow$ ${ }^{236} \mathrm{U}$ at low excitation energies. As a result, we found that the current model can well reproduce isotopic fission-fragment distribution which can be compared favorably with major libraries.
\end{abstract}

\section{Introduction}

In the high burn-up nuclear and light water reactors, information on minor actinides, such as $\mathrm{Cm}$ and Am, plays a key role for their safety. However, it is difficult to obtain sufficient accuracy for the concentration of these nuclei by experiments even now. Our aim is to develop a theoretical model, which can provide such high-quality data. From phenomenological models such as superposition of Gaussians to microscopic models, there are so many approaches to nuclear fission. The reasons why we select the Langevin model, are as follows. The Langevin calculations with only a few essential variables can give us an intuitive image of a fissioning nucleus. Such simplicity of the Langevin model enables us to track each physical quantities directly, and to compare them with experiments. However, in general, the Langevin models have been used at high energies where the statistical dynamics is more essential than the quantum effects. Fission reactions in nuclear reactors proceed at low energies, usually $20 \mathrm{MeV}$ at the highest. At such energies, nuclei have their own characteristics. Therefore, we cannot neglect quantum corrections, although these corrections are difficult to treat. Our previous research [1], overcame these difficulties, and successfully reproduced mass distributions of $U$ and

ae-mail: chikako@nr.titeich.ac.jp 
$\mathrm{Pu}$ at low energies. But that model is still not sufficient to apply to nuclear reactor physics. The isotope distributions are required. In this paper, we extend our model in order to treat the charge mode that allows the isotope yield calculation. Difference between the current work and other recent works relating to the fission properties based on the Langevin model [2, 3], is the way of the charge mode. We consider the charge mode simultaneously with other degrees of freedom within unified computational scheme solving a system of coupled equations. Another important feature of the present model is that the microscopic shell effects are essential part of our model that is very important for the considered energy region.

\section{Method}

We describe the shape of a compound nucleus, using the two-center shell model by three variables. They correspond to distance between the two centers of mass $z$, deformation $\delta$, and mass asymmetry $\eta_{A}$. In order to treat the isotope distributions, we introduce a new variable $\eta_{Z}$ for the charge mode. The Langevin approach treats nuclear fission as a Brownian motion of particle representing by the chosen collective variables. In the equation of motion, the dissipation term and the fluctuation term for the Brownian motion, are added.

$$
\begin{aligned}
\dot{q}_{i} & =m_{i j}^{-1} p_{j} \\
\dot{p}_{i} & =-\frac{\partial V}{\partial q_{i}}-\frac{1}{2} \frac{\partial}{\partial q_{i}} m_{j k}^{-1} p_{j} p_{k}-\gamma_{i j} m_{j k}^{-1} p_{k}+g_{i j} R_{j}(t),
\end{aligned}
$$

where $q_{i}=z, \delta, \eta_{A}$, and $\eta_{Z}(i=1,2,3,4)$ are defined as follows.

$$
z=\frac{z_{0}}{B R}, B=\frac{3+\delta}{3-2 \delta}, \delta=\frac{3(a-b)}{2 a+b}, \eta_{A}=\frac{A_{1}-A_{2}}{A_{1}+A_{2}}, \eta_{Z}=\frac{Z_{1}-Z_{2}}{Z_{1}+Z_{2}} .
$$

Here, $A_{1}, A_{2}, Z_{1}$, and $Z_{2}$ are the mass and charge numbers of fragment 1 and 2 , respectively. We assume that the deformation is common between a left and a right fragment. The random force in Eq. (1) satisfies $<R_{i}>=0$ and $\left\langle R_{i}\left(t_{1}\right) R_{j}\left(t_{2}\right)>=2 \delta_{i j}\left(t_{1}-t_{2}\right)\right.$. The Einstein relation between the diffusion coefficient $g_{i j}$ and the heat-bath temperature $T$ is $\sum_{k} g_{i k} g_{k j}=T \gamma_{i j}$, where $\gamma_{i j}$ is the friction tensor. The heat-bath temperature for the mass-mode $(i=1,2,3)$ is expressed as $T=\sqrt{E_{\text {int }} / a(\mathbf{q})}$ using the level density parameter $a(\mathbf{q})$, while we use the effective temperature $T_{Z}=\frac{\hbar \omega_{Z}}{2} \operatorname{coth} \frac{\hbar \omega_{Z}}{2 T}$ for the charge mode $(i=4)$ instead of the heat-bath temperature $T$, where $\omega_{Z}$ is the frequency with respect to $\eta_{Z}$. Here, $E_{\text {int }}$ is the intrinsic energy defined as follows.

$$
E_{\text {int }}=E^{*}-\frac{1}{2} m_{i j}^{-1} p_{i} p_{j}-V
$$

$E^{*}$ is the excited energy, and $V$ is the potential energy.

Basically, for the potential energy, we use a similar potential as in the three dimensional case [1]. The difference between the three dimensional case and the current model is the fluctuation around an expectation value of the charge asymmetry. We have solved the Langevin equations with the charge asymmetry around the expectation value by using the potential as follows. The coefficient of the fluctuation-potential term in Eq. (6) can be derived from the Harmonic approximation. Potential terms relating to the charge asymmetry, are symmetry energy and the Coulomb energy. Then we can obtain the analytical expression for the coefficient $C_{<\eta_{Z}>}$ with the assumption of homogeneous charge spheres, as derived in $[4,5]$ because

$$
C_{\eta_{Z}}=\left.\left.\frac{\partial^{2} V}{\partial \eta_{Z}^{2}}\right|_{\eta_{Z}=<\eta_{Z}>} \simeq \frac{\partial^{2} V_{S y m}}{\partial \eta_{Z}^{2}}\right|_{\eta_{Z}=<\eta_{Z}>}+\left.\frac{\partial^{2} V_{\text {Coul }}}{\partial \eta_{Z}^{2}}\right|_{\eta_{Z}=<\eta_{Z}>}
$$


The harmonic approximation of the potential is as follows.

$$
\begin{aligned}
V & =V_{L D}+\left(V_{\text {Shell }}(\mathbf{q}, T=0)+V_{\text {Pair }}(\mathbf{q}, T=0)\right) \exp \left(-\frac{a T^{2}}{E_{d}}\right)+\frac{l(l+1) \hbar^{2}}{2 I} \\
& =V\left(q_{i},<\eta_{Z}>\right)+\frac{C_{\eta_{Z}}}{2}\left(\eta_{Z}-<\eta_{Z}>\right)^{2}
\end{aligned}
$$

Here, note that the liquid-drop model (the original potential used in [1]) presumes the Unified Charge Distribution (UCD). As already discussed in early 80s [8], the expectation value of the charge asymmetry is not that of the UCD. Deviation from the UCD has been observed.

$$
\begin{aligned}
V\left(q_{i},<\eta_{Z}>_{U C D}\right) & =\left.V_{L D}\right|_{<\eta_{Z}>_{U C D}}+\left.\left(V_{S \text { hell }}+V_{\text {Pair }}\right) \exp \left(-\frac{a T^{2}}{E_{d}}\right)\right|_{<\eta_{Z}>U C D}+\left.\frac{l(l+1) \hbar^{2}}{2 I}\right|_{<\eta_{Z}>U C D} \\
& =V\left(q_{i},<\eta_{Z}>\right)+\frac{C_{\eta_{Z}}}{2}\left(<\eta_{Z}>_{U C D}-<\eta_{Z}>\right)^{2}
\end{aligned}
$$

Finally, the potential can be expressed using the potential relating to the liquid-drop potential, the charge fluctuation around the expectation value of the charge asymmetry, and and the charge deviation from the UCD,

$$
V=V\left(q_{i},<\eta_{Z}>_{U C D}\right)+\frac{C_{\eta_{Z}}}{2}\left(\eta_{Z}-<\eta_{Z}>\right)^{2}-\frac{C_{\eta_{Z}}}{2}\left(<\eta_{Z}>_{U C D}-<\eta_{Z}>\right)^{2}
$$

As we mentioned in the Introduction, in order to treat low-energy fission, we include quantum corrections in the potential. For the shell correction, we adopt the Strutinsky method from the single-particle levels of the two-center shell model. The BCS pairing effect is also included. The shell correction has a temperature dependence with the shell damping energy of the Ignatyuk's value. This modification of the potential energy changes trajectory, because the derivative of the fluctuation in terms of each variable $q_{i}$ affects the time evolution. We estimate the $\left\langle\eta_{Z}\right\rangle$ following [6]. The UCD hypothesis is defined as

$$
\begin{aligned}
\frac{Z}{A} & =\frac{Z_{H}^{U C D}}{A_{H}^{\prime}}=\frac{Z_{L}^{U C D}}{Z_{L}^{\prime}} \\
A_{H}^{\prime} & =A_{H}+v_{H}, \\
A_{L}^{\prime} & =A_{L}+v_{L}, \\
A & =A_{H}^{\prime}+A_{L}^{\prime},
\end{aligned}
$$

where the $v_{H}$ and $v_{L}$ is an averaged number of evaporated neutrons from each fragment, respectively.

Another extension of the Langevin model is the transport coefficients. For the mass mode [1], we use the same one as the three dimensional calculations. The Werner-Wheeler approximation for the inertial mass, and the Wall and Window formula for the friction tensor. For the charge mode [4, 5], we neglect the off-diagonal components. Here, note that we assume the evolution of the charge mode starts just after the neck appearance in a compound nucleus. For the mass tensor of the charge mode, we approximate a nuclear shape near the scission point as two spheres connected by a circular hole with radius $r_{N}$. The mass tensor can be described as

$$
m_{i j}=\left(\begin{array}{cccc}
m_{11} & m_{21} & m_{31} & 0 \\
m_{12} & m_{22} & m_{32} & 0 \\
m_{13} & m_{23} & m_{33} & 0 \\
0 & 0 & 0 & m_{\eta_{Z} \eta_{Z}}
\end{array}\right),
$$


where $m_{i j}$ (for $\mathrm{i}=1,2,3$ ) is a matrix element derived from Werner-Wheeler approximation. The indexes $i$ and $j=1,2,3,4$ correspond to $\delta, \eta_{A}$ and $\eta_{Z}$, respectively. The charge component of $m_{i j}$ is expressed as follows for a flow of viscous incompressible liquid through that cylindrical neck.

$$
\begin{gathered}
m_{\eta_{Z} \eta_{Z}}=\frac{m}{3 \pi \rho} \frac{Z A^{2}}{N} \frac{\left(l+2 r_{N}\right)}{r_{N}^{2}} \\
\gamma_{i j}=\left(\begin{array}{cccc}
\gamma_{11} & \gamma_{21} & \gamma_{31} & 0 \\
\gamma_{12} & \gamma_{22} & \gamma_{32} & 0 \\
\gamma_{13} & \gamma_{23} & \gamma_{33} & 0 \\
0 & 0 & 0 & \gamma_{\eta_{Z} \eta_{Z}}
\end{array}\right),
\end{gathered}
$$

where the friction parameter for the charge mode,

$$
\gamma_{\eta_{Z} \eta_{Z}}=\frac{4 m}{9 \rho} \frac{A Z}{N}\left[N \overline{v_{p}}+Z \overline{v_{n}}\right] \frac{1}{\Delta \sigma},
$$

is derived in $[4,5]$ from the one-body dissipation model.

\section{Results and Analysis}

In this section, we show various properties of obtained fission-fragments using the Langevin model with the charge fluctuation for ${ }^{236} \mathrm{U}$ at $E *=20 \mathrm{MeV}$ which corresponds to $n+{ }^{235} \mathrm{U}$ at $E_{n}=14 \mathrm{MeV}$. The current mass distribution reproduces the peak positions and width of the experimental data as well as the result only with the mass mode. It means that the current modification (including the charge mode simultaneously with the mass mode) keeps the gross feature of the fissioning system. However, the statistics of current calculation is not sufficient compared to the three dimensional case.

Fig. 1 is the JENDL data [7] of ${ }^{236} \mathrm{U}$ thermal fission on the nuclear chart as a reference. Compared to the JENDL data, we found that our result shown in Fig. 2 reproduce the nuclear species on the chart and yield structures, such as the peak positions, the inter-peak structure and the deviation from the UCD line. Unfortunately, it is difficult to see the tail structure in the JENDL (nuclei in blue in Fig. 1) due to the poor statistics, so we expect that it can be reproduced with enough data.

Fig. 3 to Fig. 5 are all isotope distribution of ${ }^{236} \mathrm{U}$ at $E *=20 \mathrm{MeV}$. As seen in Fig. 2, the current fission-fragments have narrow distribution width, compared to the experimental data. As a result, the minimum number in the the isotope distribution is larger than the major libraries, while the maximum proton is smaller than them, as seen in Fig. 3) and Fig. 4. However, the peak position and the curvature of our results can reproduce the experimental values, qualitatively and quantitatively.

Symbols are common in Fig. 3 to Fig. 5. Red line with filled circle is our calculation on a log scale. Black line with open circle is the JENDL/FPY-2011 [7] for ${ }^{236}$ U thermal-fission. Blue dashedline with open square is the JEFF-3.1.1 [11] for ${ }^{236} \mathrm{U}$ thermal-fission. Purple dashed-line with open triangle is the ENDF/B-VII [12] for ${ }^{236} \mathrm{U}$ thermal fission. Green dashed-line with open invertedtriangle is the GEF [10] for ${ }^{236} \mathrm{U}$ thermal-fission, respectively.

We found that our obtained nuclei with enough yields can reproduce major libraries very well in various mass numbers as shown in Fig. 3 and Fig. 4. Such characteristics of the calculated isotope distribution can be commonly seen in all data between $Z=39$ to $Z=52$. Fig. 5 is the same as the previous two figures but for the specific mass number $A=132$. Even for the fixed mass number, such consistency with the major libraries can be also found. 


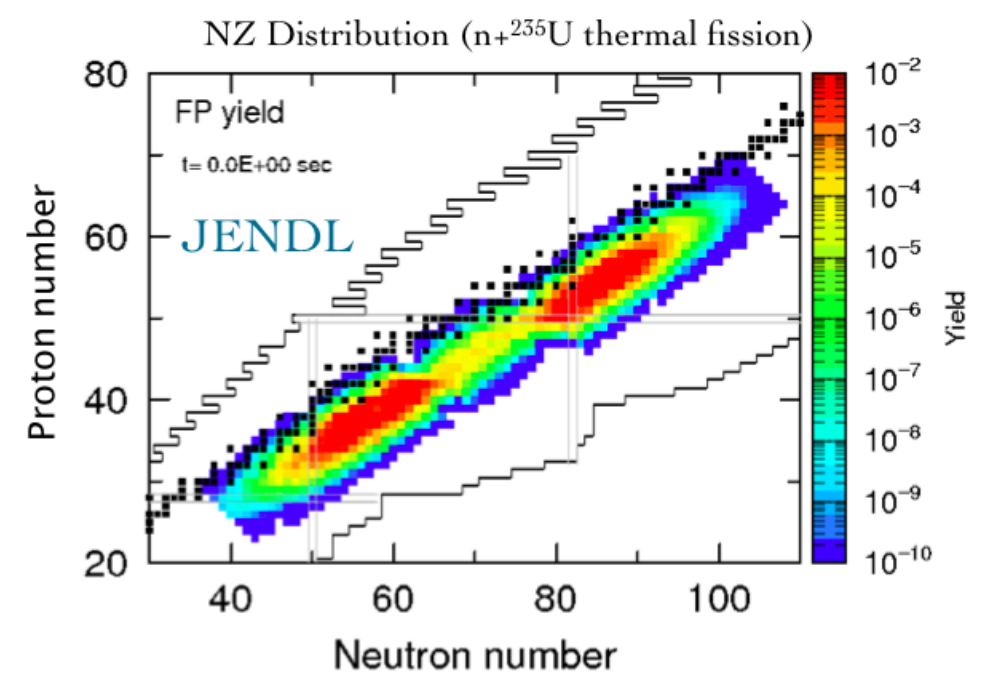

Figure 1. The JENDL data for ${ }^{236} \mathrm{U}$ thermal-fission is plotted in the nuclear chart. Fragment yield is shown in color map.

\section{Summary}

In this paper, we extended our Langevin model based on the two-center shell-model parametrization by including the contribution from the fluctuation around the most stable charge asymmetry. We performed the Langevin calculation for the compound system of ${ }^{236} \mathrm{U}$ at $E *=20 \mathrm{MeV}$ in terms of shape parameters, the mass asymmetry and the charge asymmetry simultaneously. We assumed the expectation value for the charge asymmetry including the charge deviation from the UCD as defined in [6]. As a result, our calculations showed good agreement with the NZ-distributions and isotope distributions of the major libraries, and that our model can provide sufficient accuracy to be compared to them, without any modification.

\section{References}

[1] Y. Aritomo and S. Chiba, PRC 88 (2013) 044614 and references therein.

[2] K. Mazurek, C. Schmitt and P. N. Nadtochy, PRC 91 (2015) 041603(R).

[3] M. R. Pahlavani and S. M, Mirfathi, PRC 92 (2015) 024622.

[4] A. V. Karpov and G. D. Adeev, EPJA 14 (2002) 169 and references therein.

[5] A. V. Karpov and G. D. Adeev, Phys. At. Nucl. 65 (2002) 1596.

[6] G. D. Adeev, Fiz. Elem. Chastis At. Yadra 23 (1992) 1572.

[7] K. Shibata, O. Iwamoto, T. Nakagawa, N. Iwamoto, A. Ichihara, S. Kunieda, S. Chiba, K. Furutaka, N. Otuka, T. Ohsawa, T. Murata, H. Matsunobu, A. Zukeran, S. Kamada, and J. Katakura, J. Nucl. Sci. Technol. 48 (2011) 1.

[8] R. K. Gaputa and D. R. Saroha, PRC 30 (1984) 395 and reference therein.

[9] A. C. Wahl, Atomic Data and Nucl. Data Tables 39 (1988) 1.

[10] K.-H. Schmidt, B. Jurado, Ch. Amouroux, JEFF-Report 24 (2014) 1. 


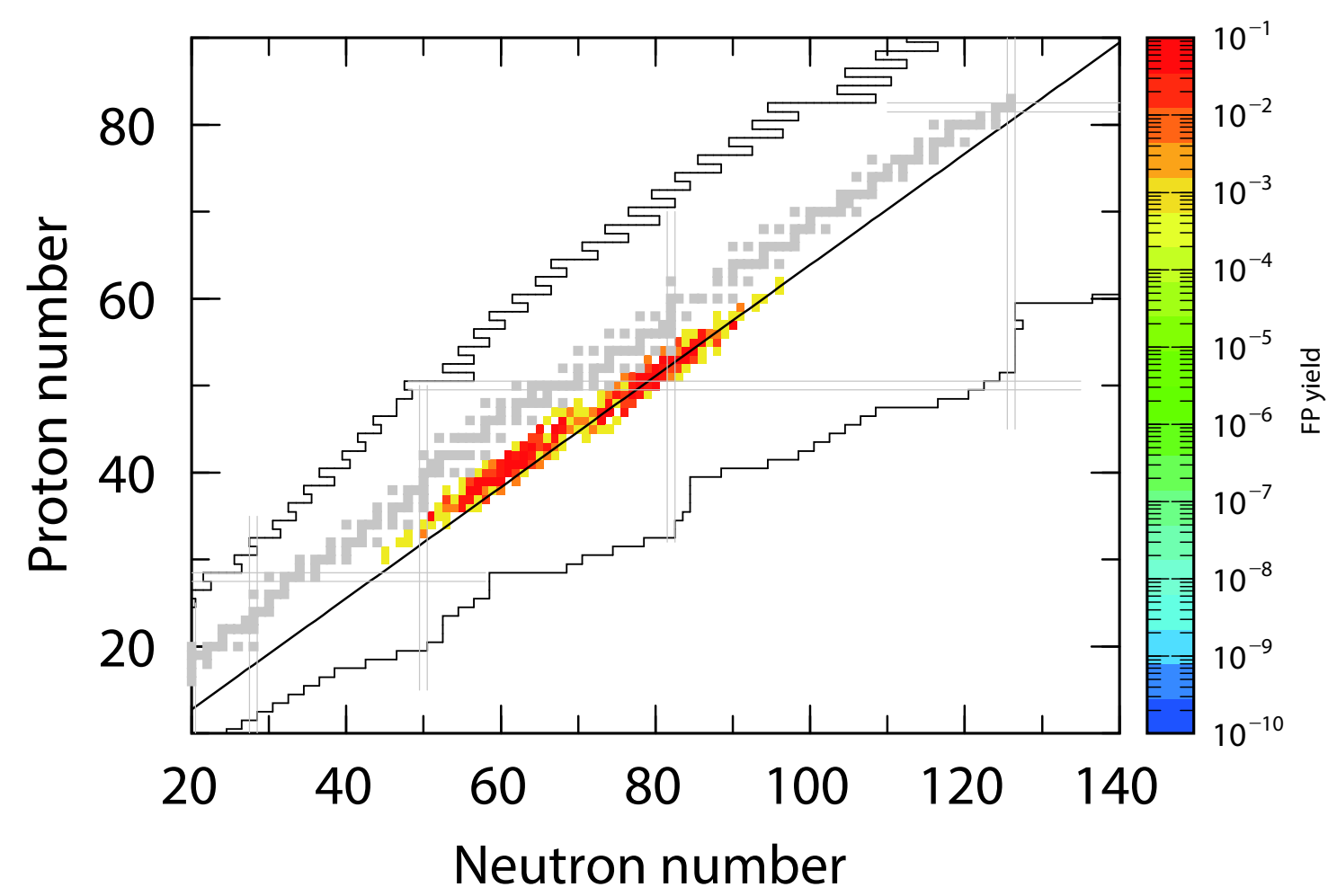

Figure 2. Calculated fragment yields in color map. The 1.5 neutrons are removed from each fragment as emitted prompt neutrons.

[11] A. Santamarina et al., JEFF-Report 22 (2009) 1.

[12] M.B. Chadwick et al., Nucl. Data Sheets 112 (2011) 2887. 


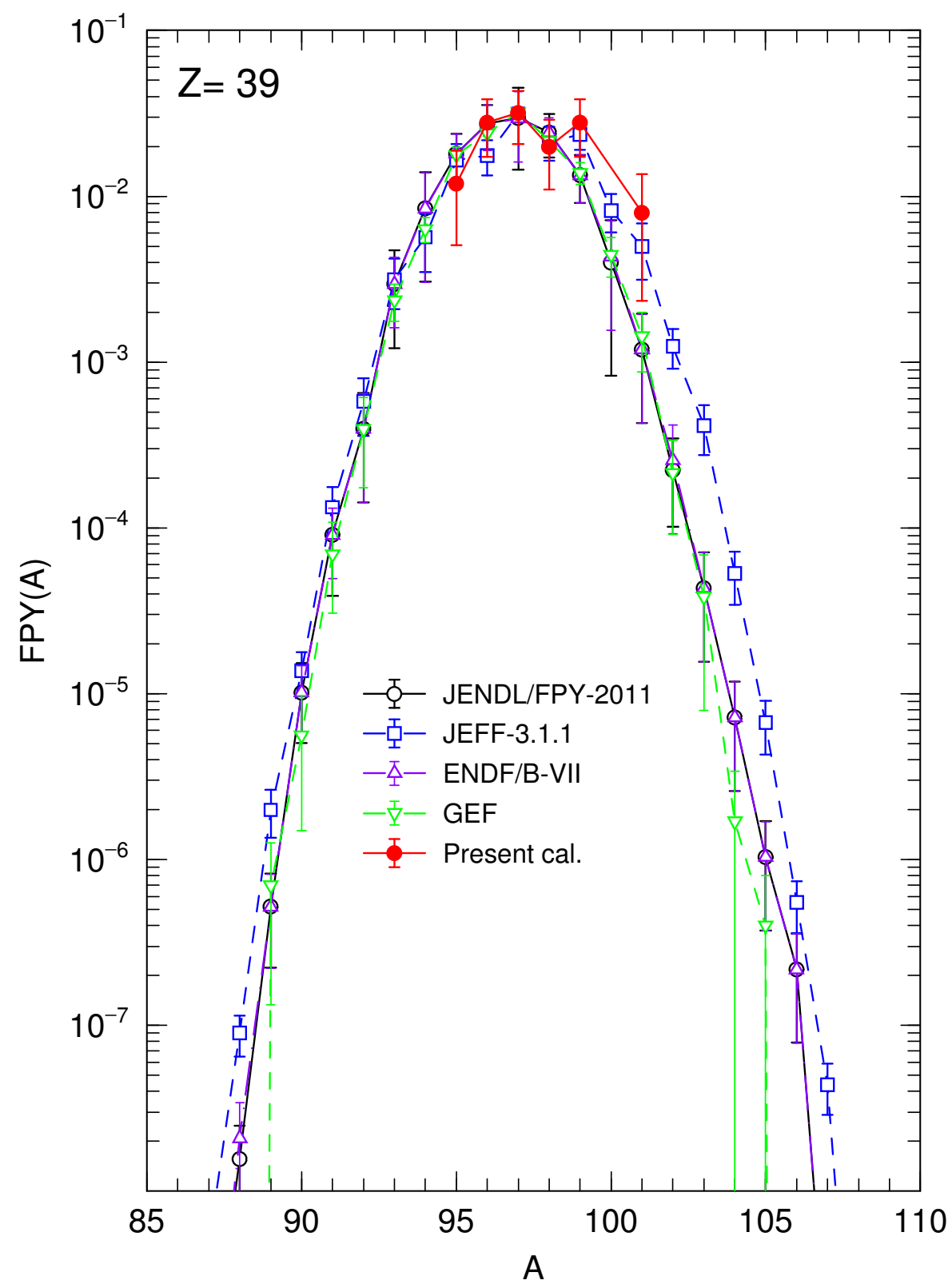

Figure 3. Isotope distribution as a function of mass number $A$ is shown, when $Z=39$, with major libraries. Red line with filled circle is our calculation on a log scale. Black line with open circle is the JENDL/FPY-2011 [7] for ${ }^{236} \mathrm{U}$ thermal-fission. Blue dashed-line with open square is the JEFF-3.1.1 [11] for ${ }^{236} \mathrm{U}$ thermal-fission. Purple dashed-line with open triangle is the ENDF/B-VII [12] for ${ }^{236} \mathrm{U}$ thermal fission. Green dashed-line with open inverted-triangle is the GEF [10] for ${ }^{236} \mathrm{U}$ thermal-fission. 


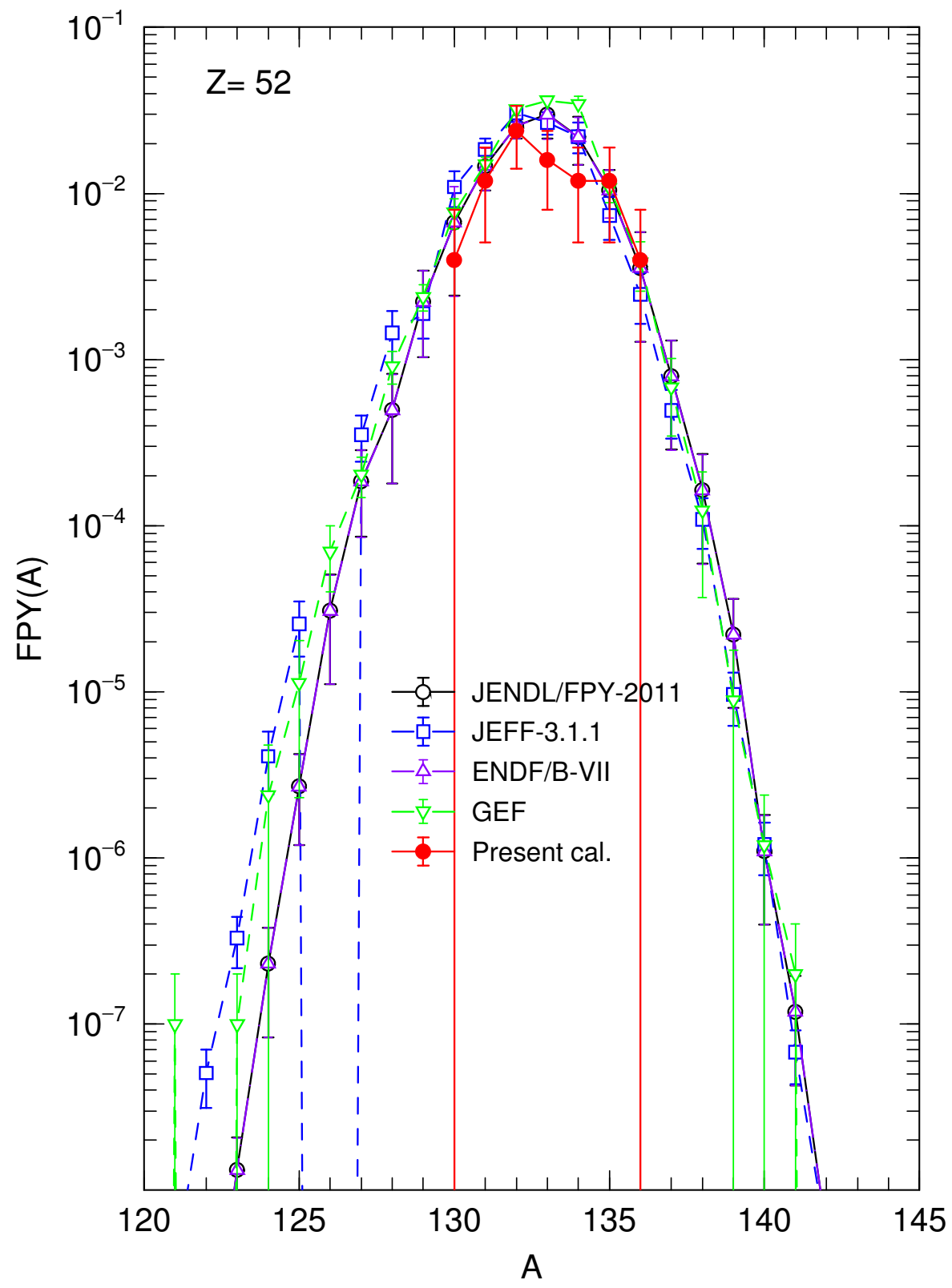

Figure 4. Isotope distribution as a function of mass number $A$ is shown, when $Z=52$, with major libraries. Red line with filled circle is our calculation on a $\log$ scale. Black line with open circle is the JENDL/FPY-2011 [7] for ${ }^{236} \mathrm{U}$ thermal-fission. Blue dashed-line with open square is the JEFF-3.1.1 [11] for ${ }^{236} \mathrm{U}$ thermal-fission. Purple dashed-line with open triangle is the ENDF/B-VII [12] for ${ }^{236} \mathrm{U}$ thermal fission. Green dashed-line with open inverted-triangle is the GEF [10] for ${ }^{236} \mathrm{U}$ thermal-fission. 


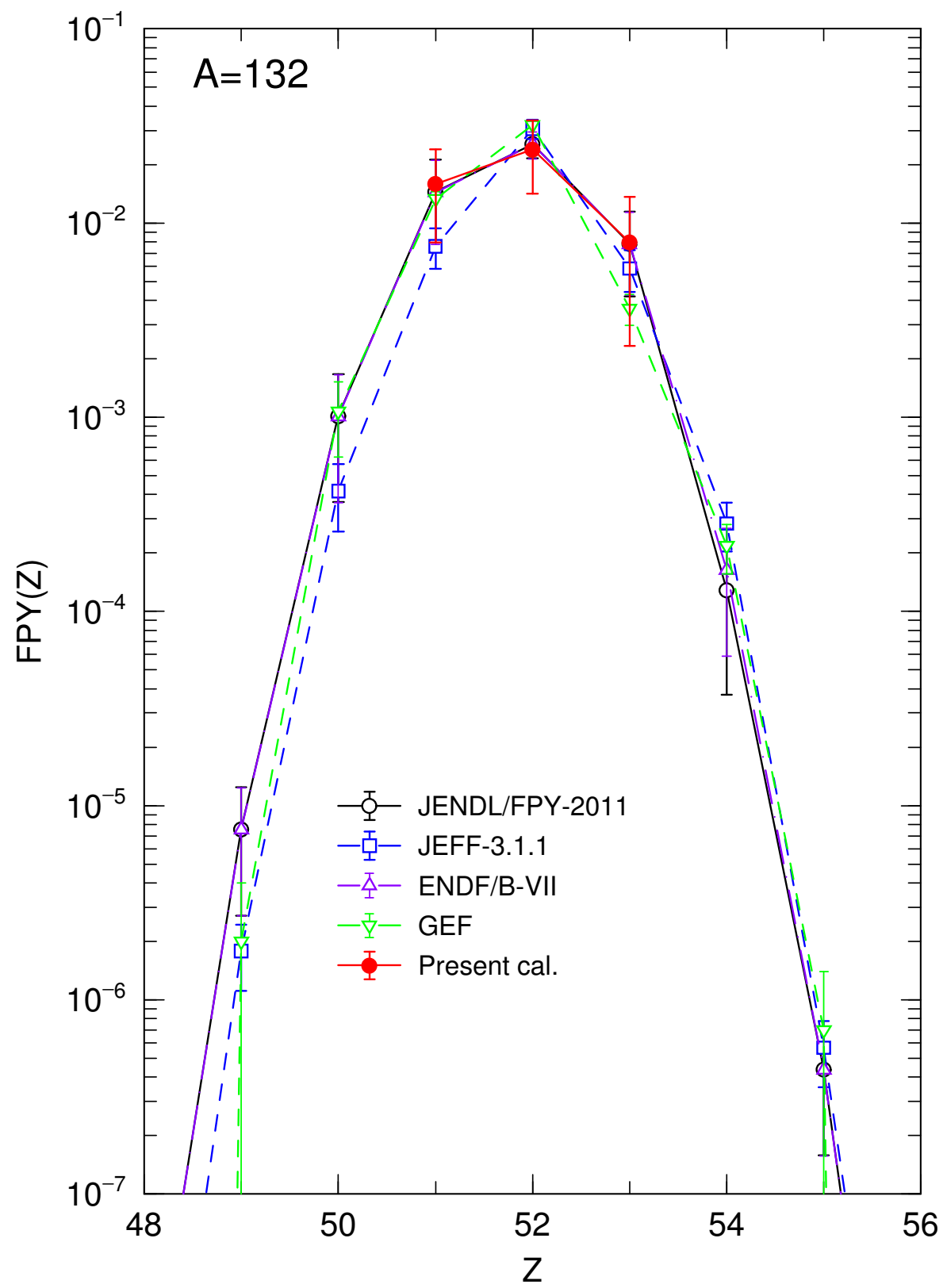

Figure 5. Isotope distribution as a function of charge number $Z$ is shown, when $A=132$, with major libraries. Red line with filled circle is our calculation on a log scale. Black line with open circle is the JENDL/FPY-2011 [7] for ${ }^{236} \mathrm{U}$ thermal-fission. Blue dashed-line with open square is the JEFF-3.1.1 [11] for ${ }^{236} \mathrm{U}$ thermal-fission. Purple dashed-line with open triangle is the ENDF/B-VII [12] for ${ }^{236} \mathrm{U}$ thermal fission. Green dashed-line with open inverted-triangle is the GEF [10] for ${ }^{236} \mathrm{U}$ thermal-fission. 